\title{
BOUNDEDNESS FOR MULTILINEAR LITTLEWOOD-PALEY OPERATORS ON TRIEBEL-LIZORKIN SPACES *
}

\author{
LIU LANZHE
}

Abstract. In this paper, we prove the boundedness in the context of Triebel-Lizorkin spaces for multilinear Littlewood-Paley operators.

1. Introduction and results. Let $\delta>0$ and $\psi$ be a function on $R^{n}$ which satisfies the following properties:

(1) $\int_{R^{n}} \psi(x) d x=0$,

(2) $|\psi(x)| \leq C(1+|x|)^{-(n+1-\delta)}$,

(3) $|\psi(x+y)-\psi(x)| \leq C|y|(1+|x|)^{-(n+2-\delta)}$ when $2|y|<|x|$.

Fixed $\mu>1$. Let $m$ be a positive integer and $A$ be a function on $R^{n}$. The multilinear Littlewood-Paley operator is defined by

$$
g_{\mu}^{A}(f)(x)=\left[\iint_{R_{+}^{n+1}}\left(\frac{t}{t+|x-y|}\right)^{n \mu}\left|F_{t}^{A}(f)(x, y)\right|^{2} \frac{d y d t}{t^{n+1}}\right]^{1 / 2},
$$

where

$$
\begin{aligned}
& F_{t}^{A}(f)(x, y)=\int_{R^{n}} \frac{R_{m+1}(A ; x, z)}{|x-z|^{m}} f(z) \psi_{t}(y-z) d z \\
& R_{m+1}(A ; x, y)=A(x)-\sum_{|\alpha| \leq m} \frac{1}{\alpha !} D^{\alpha} A(y)(x-y)^{\alpha},
\end{aligned}
$$

and $\psi_{t}(x)=t^{-n+\delta} \psi(x / t)$ for $t>0$. Let $F_{t}(f)(y)=f * \psi_{t}(y)$. We also define that

$$
g_{\mu}(f)(x)=\left(\iint_{R_{+}^{n+1}}\left(\frac{t}{t+|x-y|}\right)^{n \mu}\left|F_{t}(f)(y)\right|^{2} \frac{d y d t}{t^{n+1}}\right)^{1 / 2},
$$

which is the Littlewood-Paley operator (see [18]).

Let $H$ be the Hilbert space $H=\left\{h:\|h\|=\left(\iint_{R_{+}^{n+1}}|h(t)|^{2} d y d t / t^{n+1}\right)^{1 / 2}<\infty\right\}$. Then for each fixed $x \in R^{n}, F_{t}^{A}(f)(x, y)$ may be viewed as a mapping from $(0,+\infty)$ to $H$, and it is clear that

$$
\begin{aligned}
& g_{\mu}^{A}(f)(x)=\left\|\left(\frac{t}{t+|x-y|}\right)^{n \mu / 2} F_{t}^{A}(f)(x, y)\right\|, \\
& g_{\mu}(f)(x)=\left\|\left(\frac{t}{t+|x-y|}\right)^{n \mu / 2} F_{t}(f)(y)\right\| .
\end{aligned}
$$

\footnotetext{
${ }^{*}$ Received March 23, 2003; accepted for publication February 3, 2004. Supported by the NNSF (Grant: 10271071).

${ }^{\dagger}$ College of Mathematics and Computer, Changsha University of Science and Technology, Changsha 410077, P.R. of China (lanzheliu@263.net).
} 
Note that when $m=0, g_{\mu}^{A}$ is just the commutator of Littlewood-Paley operator (see [1][13-16]). It is well known that multilinear operators are of great interest in harmonic analysis and have been widely studied by many authors (see [3-6][9][10]). In [11][17], Janson and Paluszynski obtain the boundedness of commutators generated by the Calderón-Zygmund operator or fractional integral operator with Lipschitz functions on Triebel-Lizorkin spaces. The main purpose of this paper is to discuss the boundedness of the multilinear Littlewood-Paley operators in the context of TriebelLizorkin spaces. First, let us introduce some notation. We will work on $R^{n}, n>1$. Throughout this paper, $M(f)$ will denote the Hardy-littlewood maximal function of $f, Q$ will denote a cube of $R^{n}$ with side parallel to the axes, and for a cube $Q$, let $f_{Q}=|Q|^{-1} \int_{Q} f(x) d x$ and $f^{\#}(x)=\sup _{x \in Q}|Q|^{-1} \int_{Q}\left|f(y)-f_{Q}\right| d y$. For $\beta>0$ and $p>1$, let $\dot{F}_{p}^{\beta, \infty}$ be the homogeneous Triebel-Lizorkin space. The Lipschitz space $\dot{\Lambda}_{\beta}$ is the space of functions $f$ such that

$$
\|f\|_{\dot{\lambda}_{\beta}}=\sup _{\substack{x, h \in R^{n} \\ h \neq 0}}\left|\Delta_{h}^{[\beta]+1} f(x)\right| /|h|^{\beta}<\infty
$$

where $\Delta_{h}^{k}$ denotes the $k$-th difference operator (see[17]).

We shall prove the following theorems in Section 3.

TheOrem 1. Let $0 \leq \delta<n, 0<\beta<1 / 2,1<p<n / \delta, 1 / p-1 / q=\delta / n$ and $D^{\alpha} A \in \dot{\Lambda}_{\beta}$ for $|\alpha|=m$. Then $g_{\mu}^{A}$ is bounded from $L^{p}\left(R^{n}\right)$ to $\dot{F}_{q}^{\beta, \infty}\left(R^{n}\right)$.

TheOREM 2. Let $0 \leq \delta<n, 0<\beta<1 / 2,1<p<n /(\delta+\beta), 1 / p-1 / q=$ $(\delta+\beta) / n$ and $D^{\alpha} A \in \dot{\Lambda}_{\beta}$ for $|\alpha|=m$. Then $g_{\mu}^{A}$ is bounded from $L^{p}\left(R^{n}\right)$ to $L^{q}\left(R^{n}\right)$.

Theorem 3. Let $0 \leq \delta<n, 0<\beta<1 / 2, \delta+\beta<n$ and $D^{\alpha} A \in \dot{\Lambda}_{\beta}$ for $|\alpha|=m$. Then for any $\lambda>0$,

$$
\left|\left\{x \in R^{n}: g_{\mu}^{A}(f)(x)>\lambda\right\}\right| \leq C\left(\sum_{|\alpha|=m}\left\|D^{\alpha} A\right\|_{\dot{\lambda}_{\beta}}\|f\|_{L^{1}} / \lambda\right)^{n /(n-\delta-\beta)} .
$$

2. Some lemmas. We begin with some preliminary lemmas.

LEMma 1 (see [17]). For $0<\beta<1,1<p<\infty$, we have

$$
\begin{aligned}
\|f\|_{\dot{F}_{p}^{\beta, \infty}} & \approx\left\|\sup _{Q} \frac{1}{|Q|^{1+\beta / n}} \int_{Q}\left|f(x)-f_{Q}\right| d x \mid\right\|_{L^{p}} \\
& \approx\left\|\sup _{\cdot \in Q} \inf _{c} \frac{1}{|Q|^{1+\beta / n}} \int_{Q}|f(x)-c| d x\right\|_{L^{p}} .
\end{aligned}
$$

Lemma 2 (see [17]). For $0<\beta<1,1 \leq p \leq \infty$, we have

$$
\|f\|_{\wedge_{\beta}} \approx \sup _{Q} \frac{1}{|Q|^{1+\beta / n}} \int_{Q}\left|f(x)-f_{Q}\right| d x \approx \sup _{Q} \frac{1}{|Q|^{\beta / n}}\left(\frac{1}{|Q|} \int_{Q}\left|f(x)-f_{Q}\right|^{p} d x\right)^{1 / p} .
$$

Lemma 3 (see [2]). For $1 \leq r<\infty$ and $0<\delta<n$, let

$$
M_{\delta, r}(f)(x)=\sup _{x \in Q}\left(\frac{1}{|Q|^{1-\delta r / n}} \int_{Q}|f(y)|^{r} d y\right)^{1 / r} .
$$


Suppose that $r<p<n / \delta$ and $1 / q=1 / p-\delta / n$. Then $\left\|M_{\delta, r}(f)\right\|_{L^{q}} \leq C\|f\|_{L^{p}}$.

Lemma 4 (see [8]). Let $Q_{1} \subset Q_{2}$. Then

$$
\left|f_{Q_{1}}-f_{Q_{2}}\right| \leq C|| f \|_{{\dot{\lambda_{\beta}}}_{\beta}}\left|Q_{2}\right|^{\beta / n} \text {. }
$$

Lemma 5 (see [6]). Let $A$ be a function on $R^{n}$ and $D^{\alpha} A \in L^{q}\left(R^{n}\right)$ for $|\alpha|=m$ and some $q>n$. Then

$$
\left|R_{m}(A ; x, y)\right| \leq C|x-y|^{m} \sum_{|\alpha|=m}\left(\frac{1}{|\tilde{Q}(x, y)|} \int_{\tilde{Q}(x, y)}\left|D^{\alpha} A(z)\right|^{q} d z\right)^{1 / q}
$$

where $\tilde{Q}(x, y)$ is the cube centered at $x$ and having side length $5 \sqrt{n}|x-y|$.

Lemma 6. Let $0 \leq \delta<n, 1<p<\infty$ and $D^{\alpha} A \in \dot{\Lambda}_{\beta}$ for $|\alpha|=m, 1<r \leq \infty$, $1 / q=1 / p+1 / r-\delta / n$. Then $g_{\mu}^{A}$ is bounded from $L^{p}\left(R^{n}\right)$ to $L^{q}\left(R^{n}\right)$, that is

$$
\left\|g_{\mu}^{A}(f)\right\|_{L^{q}} \leq C \sum_{|\alpha|=m}\left\|D^{\alpha} A\right\|{\dot{\dot{\wedge}_{\beta}}}\|f\|_{L^{p}}
$$

Proof. By the Minkowski' inequality and the condition of $\psi$, we have

$$
\begin{aligned}
& g_{\mu}^{A}(f)(x) \\
\leq & \int_{R^{n}} \frac{|f(z)|\left|R_{m+1}(A ; x, z)\right|}{|x-z|^{m}}\left(\int_{R_{+}^{n+1}}\left(\frac{t}{t+|x-y|}\right)^{n \mu}\left|\psi_{t}(y-z)\right|^{2} \frac{d y d t}{t^{1+n}}\right)^{1 / 2} d z \\
\leq & C \int_{R^{n}} \frac{|f(z)|\left|R_{m+1}(A ; x, z)\right|}{|x-z|^{m}} \\
& \times\left(\int_{0}^{\infty} \int_{R^{n}}\left(\frac{t}{t+|x-y|}\right)^{n \mu} \frac{t^{-2 n+2 \delta}}{(1+|y-z| / t)^{2 n+2-2 \delta}} \frac{d y d t}{t^{1+n}}\right)^{1 / 2} d z \\
\leq & C \int_{R^{n}} \frac{|f(z)|\left|R_{m+1}(A ; x, z)\right|}{|x-z|^{m}} \\
& \times\left[\int_{0}^{\infty}\left(t^{-n} \int_{R^{n}}\left(\frac{t}{t+|x-y|}\right)^{n \mu} \frac{d y}{(t+|y-z|)^{2 n+2-2 \delta}}\right) t d t\right]^{1 / 2} d z .
\end{aligned}
$$

Noting that

$$
\begin{aligned}
t^{-n} \int_{R^{n}}\left(\frac{t}{t+|x-y|}\right)^{n \mu} \frac{d y}{(t+|y-z|)^{2 n+2-2 \delta}} & \leq C M\left(\frac{1}{(t+|x-z|)^{2 n+2-2 \delta}}\right) \\
& \leq \frac{C}{(t+|x-z|)^{2 n+2-2 \delta}}
\end{aligned}
$$

and

$$
\int_{0}^{\infty} \frac{t d t}{(t+|x-z|)^{2 n+2-2 \delta}}=C|x-z|^{-2 n+2 \delta}
$$

we obtain

$$
\begin{aligned}
g_{\mu}^{A}(f)(x) & \leq C \int_{R^{n}} \frac{|f(z)|\left|R_{m+1}(A ; x, z)\right|}{|x-z|^{m}}\left(\int_{0}^{\infty} \frac{t d t}{(t+|x-z|)^{2 n+2-2 \delta}}\right)^{1 / 2} d z \\
& =C \int_{R^{n}} \frac{|f(z)|\left|R_{m+1}(A ; x, z)\right|}{|x-z|^{m+n-\delta}} d z
\end{aligned}
$$

thus, the lemma follows from [3]. 


\section{Proof of theorems.}

Proof of Theorem 1. Fix a cube $Q=Q\left(x_{0}, l\right)$ and $\tilde{x} \in Q$. Let $\tilde{Q}=5 \sqrt{n} Q$ and $\tilde{A}(x)=A(x)-\sum_{|\alpha|=m} \frac{1}{\alpha !}\left(D^{\alpha} A\right)_{\tilde{Q}} x^{\alpha}$, then $R_{m}(A ; x, y)=R_{m}(\tilde{A} ; x, y)$ and $D^{\alpha} \tilde{A}=$ $D^{\alpha} A-\left(D^{\alpha} A\right)_{\tilde{Q}}$ for $|\alpha|=m$. We write, for $f_{1}=f \chi_{\tilde{Q}}$ and $f_{2}=f \chi_{R^{n} \backslash \tilde{Q}}$,

$$
\begin{aligned}
F_{t}^{A}(f)(x, y)= & \int_{R^{n}} \frac{R_{m+1}(\tilde{A} ; x, z)}{|x-z|^{m}} \psi_{t}(y-z) f(z) d z \\
= & \int_{R^{n}} \frac{R_{m+1}(\tilde{A} ; x, z)}{|x-z|^{m}} \psi_{t}(y-z) f_{2}(z) d z+\int_{R^{n}} \frac{R_{m}(\tilde{A} ; x, z)}{|x-z|^{m}} \psi_{t}(y-z) f_{1}(z) d z \\
& -\sum_{|\alpha|=m} \frac{1}{\alpha !} \int_{R^{n}} \frac{(x-z)^{\alpha} \psi_{t}(y-z)}{|x-z|^{m}} D^{\alpha} \tilde{A}(z) f_{1}(z) d z
\end{aligned}
$$

then

$$
\begin{aligned}
& \left|g_{\mu}^{A}(f)(x)-g_{\mu}^{\tilde{A}}\left(f_{2}\right)\left(x_{0}\right)\right| \\
= & \left\|\left(\frac{t}{t+|x-y|}\right)^{n \mu / 2} F_{t}^{A}(f)(x, y)\right\|-\left\|\left(\frac{t}{t+\left|x_{0}-y\right|}\right)^{n \mu / 2} F_{t}^{\tilde{A}}\left(f_{2}\right)\left(x_{0}, y\right)\right\| \\
\leq & \left\|\left(\frac{t}{t+|x-y|}\right)^{n \mu / 2} F_{t}\left(\frac{R_{m}(\tilde{A} ; x, \cdot)}{|x-\cdot|^{m}} f_{1}\right)(y)\right\| \\
& +\sum_{|\alpha|=m} \frac{1}{\alpha !}\left\|\left(\frac{t}{t+|x-y|}\right)^{n \mu / 2} F_{t}\left(\frac{(x-\cdot)^{\alpha}}{|x-|^{m}} D^{\alpha} \tilde{A} f_{1}\right)(y)\right\| \\
& +\left\|\left(\frac{t}{t+|x-y|}\right)^{n \mu / 2} F_{t}^{\tilde{A}}\left(f_{2}\right)(x, y)-\left(\frac{t}{t+\left|x_{0}-y\right|}\right)^{n \mu / 2} F_{t}^{\tilde{A}}\left(f_{2}\right)\left(x_{0}, y\right)\right\| \\
:= & A(x)+B(x)+C(x),
\end{aligned}
$$

thus,

$$
\begin{aligned}
& \frac{1}{|Q|^{1+\beta / n}} \int_{Q}\left|g_{\mu}^{A}(f)(x)-g_{\mu}^{\tilde{A}}\left(f_{2}\right)\left(x_{0}\right)\right| d x \\
\leq & \frac{1}{|Q|^{1+\beta / n}} \int_{Q} A(x) d x+\frac{1}{|Q|^{1+\beta / n}} \int_{Q} B(x) d x+\frac{1}{|Q|^{1+\beta / n}} \int_{Q} C(x) d x \\
:= & I+I I+I I I .
\end{aligned}
$$

Now, let us estimate $I, I I$ and $I I I$, respectively. First, for $x \in Q$ and $z \in \tilde{Q}$, using Lemma 2 and Lemma 5, we get

$$
\begin{aligned}
\left|R_{m}(\tilde{A} ; x, z)\right| & \leq C|x-z|^{m} \sum_{|\alpha|=m} \sup _{x \in \tilde{Q}}\left|D^{\alpha} A(x)-\left(D^{\alpha} A\right)_{\tilde{Q}}\right| \\
& \leq C|x-z|^{m}|Q|^{\beta / n} \sum_{|\alpha|=m}\left\|D^{\alpha} A\right\|_{\dot{\lambda}_{\beta}},
\end{aligned}
$$


thus, taking $r, s$ such that $1<r<p$ and $1 / s=1 / r-\delta / n$, by the $\left(L^{r}, L^{s}\right)$-boundedness of $g_{\mu}$ (see Lemma 6$)$ and the Hölder'inequality, we obtain

$$
\begin{aligned}
I & \leq C \sum_{|\alpha|=m}\left\|D^{\alpha} A\right\|\left\|_{\dot{\lambda}_{\beta}} \frac{1}{|Q|} \int_{Q}\left|g_{\mu}\left(f_{1}\right)(x)\right| d x \leq C \sum_{|\alpha|=m}\right\| D^{\alpha} A\left\|_{\dot{\lambda}_{\beta}}|| g_{\mu}\left(f_{1}\right)\right\|_{L^{s}}|Q|^{-1 / s} \\
& \leq\left. C \sum_{|\alpha|=m}\left\|D^{\alpha} A\right\|\right|_{\dot{\lambda}_{\beta}}\left\|f_{1}\right\|_{L^{r}}|Q|^{-1 / s} \leq C \sum_{|\alpha|=m}\left\|D^{\alpha} A\right\|_{\dot{\lambda}_{\beta}}\left(\frac{1}{|\tilde{Q}|^{1-r \delta / n}} \int_{\tilde{Q}}|f(y)|^{r} d y\right)^{1 / r} \\
& \leq C \sum_{|\alpha|=m}\left\|D^{\alpha} A\right\|_{\dot{\lambda}_{\beta}} M_{\delta, r}(f)(\tilde{x}) .
\end{aligned}
$$

Secondly, using the following inequality(see [17]):

$$
\left\|\left(D^{\alpha} A-\left(D^{\alpha} A\right)_{\tilde{Q}}\right) f \chi_{\tilde{Q}}\right\|_{L^{r}} \leq C|Q|^{1 / s+\beta / n}\left\|D^{\alpha} A\right\|_{\dot{\wedge}_{\beta}} M_{\delta, r}(f),
$$

and similar to the proof of $I$, we gain

$$
\begin{aligned}
I I & \leq \frac{C}{|Q|^{1+\beta / n}} \sum_{|\alpha|=m}\left\|g_{\mu}\left(\left(D^{\alpha} A-\left(D^{\alpha} A\right)_{\tilde{Q}}\right) f_{1}\right)\right\|_{L^{s}}|Q|^{1-1 / s} \\
& \leq C|Q|^{-\beta / n-1 / r} \sum_{|\alpha|=m}\left\|\left(D^{\alpha} A-\left(D^{\alpha} A\right)_{\tilde{Q}}\right) f_{1}\right\|_{L^{r}} \\
& \leq C \sum_{|\alpha|=m}\left\|D^{\alpha} A\right\|_{\dot{\wedge}_{\beta}} M_{\delta, r}(f)(\tilde{x}) .
\end{aligned}
$$

To estimate $I I I$, we write

$$
\begin{aligned}
& \left(\frac{t}{t+|x-y|}\right)^{n \mu / 2} F_{t}^{\tilde{A}}\left(f_{2}\right)(x, y)-\left(\frac{t}{t+\left|x_{0}-y\right|}\right)^{n \mu / 2} F_{t}^{\tilde{A}}\left(f_{2}\right)\left(x_{0}, y\right) \\
= & \int_{R^{n}}\left(\frac{t}{t+|x-y|}\right)^{n \mu / 2}\left[\frac{1}{|x-z|^{m}}-\frac{1}{\left|x_{0}-z\right|^{m}}\right] R_{m}(\tilde{A} ; x, z) \psi_{t}(y-z) f_{2}(z) d z \\
& +\int_{R^{n}}\left(\frac{t}{t+|x-y|}\right)^{n \mu / 2} \frac{\psi_{t}(y-z) f_{2}(z)}{\left|x_{0}-z\right|^{m}}\left[R_{m}(\tilde{A} ; x, z)-R_{m}\left(\tilde{A} ; x_{0}, z\right)\right] d z \\
& +\int_{R^{n}}\left[\left(\frac{t}{t+|x-y|}\right)^{n \mu / 2}-\left(\frac{t}{t+\left|x_{0}-y\right|}\right)^{n \mu / 2}\right] \frac{R_{m}\left(\tilde{A} ; x_{0}, z\right) \psi_{t}(y-z) f_{2}(z)}{\left|x_{0}-z\right|^{m}} d z \\
& -\sum_{|\alpha|=m} \frac{1}{\alpha !} \int_{R^{n}}\left[\left(\frac{t}{t+|x-y|}\right)^{n \mu / 2} \frac{(x-z)^{\alpha}}{|x-z|^{m}}-\left(\frac{\left.t x_{0}-z\right)^{\alpha}}{t+\left|x_{0}-y\right|}\right)^{|x-z|^{m}}\right] \\
& \times D^{\alpha} \tilde{A}(z) \psi_{t}(y-z) f_{2}(z) d z \\
:= & I I I_{1}+I I I_{2}+I I I_{3}+I I I_{4} .
\end{aligned}
$$


Note that $|x-z| \sim\left|x_{0}-z\right|$ for $x \in Q$ and $z \in R^{n} \backslash \tilde{Q}$. By the condition of $\psi$ and similar to the proof of Lemma 6 , we obtain

$$
\begin{aligned}
& \frac{1}{|Q|^{1+\beta / n}} \int_{Q}\left\|I I I_{1}\right\| d x \\
\leq & \frac{C}{|Q|^{1+\beta / n}} \int_{Q}\left(\int_{R^{n} \backslash \tilde{Q}} \frac{\left|x-x_{0}\right|}{\left|x_{0}-z\right|^{m+n+1-\delta}}\left|R_{m}(\tilde{A} ; x, z)\right||f(z)| d z\right) d x \\
\leq & C \sum_{|\alpha|=m}\left\|D^{\alpha} A\right\|_{\dot{\lambda}_{\beta}} \sum_{k=0}^{\infty} \int_{2^{k+1} \tilde{Q} \backslash 2^{k+1} \tilde{Q}} \frac{\left|x-x_{0}\right|}{\left|x_{0}-z\right|^{n+1-\delta}}|f(z)| d z \\
\leq & C \sum_{|\alpha|=m}\left\|D^{\alpha} A\right\|_{\dot{\lambda}_{\beta}} \sum_{k=1}^{\infty} 2^{-k} \frac{1}{\left|2^{k} \tilde{Q}\right|^{1-\delta / n}} \int_{2^{k} \tilde{Q}}|f(z)| d z \\
\leq & C \sum_{|\alpha|=m}\left\|D^{\alpha} A\right\|_{\dot{\lambda}_{\beta}} \sum_{k=1}^{\infty} 2^{-k} M_{\delta, 1}(f)(\tilde{x}) \\
\leq & C \sum_{|\alpha|=m}\left\|D^{\alpha} A\right\|_{\dot{\lambda}_{\beta}} M_{\delta, 1}(f)(\tilde{x}) .
\end{aligned}
$$

For $I I I_{2}$, by the formula (see $\left.[6]\right)$ :

$$
R_{m}(\tilde{A} ; x, z)-R_{m}\left(\tilde{A} ; x_{0}, z\right)=\sum_{|\eta|<m} \frac{1}{\eta !} R_{m-|\eta|}\left(D^{\eta} \tilde{A} ; x, x_{0}\right)(x-z)^{\eta}
$$

and Lemma 5, we get

$$
\left|R_{m}(\tilde{A} ; x, z)-R_{m}\left(\tilde{A} ; x_{0}, z\right)\right| \leq C \sum_{|\alpha|=m}|| D^{\alpha} A \|_{{\dot{\lambda}}}|Q|^{\beta / n}\left|x-x_{0}\right|\left|x_{0}-z\right|^{m-1},
$$

thus

$$
\begin{aligned}
& \frac{1}{|Q|^{1+\beta / n}} \int_{Q} \| I I I_{2}|| d x \\
\leq & C \frac{1}{|Q|^{1+\beta / n}} \int_{Q} \int_{R^{n} \backslash \tilde{Q}} \frac{\left|R_{m}(\tilde{A} ; x, z)-R_{m}\left(\tilde{A} ; x_{0}, z\right)\right|}{\left|x_{0}-z\right|^{m+n-\delta}}|f(z)| d z d x \\
\leq & C \sum_{|\alpha|=m}\left\|D^{\alpha} A\right\|_{\dot{\Lambda}_{\beta}} \sum_{k=0}^{\infty} \int_{2^{k+1} \tilde{Q} \backslash 2^{k} \tilde{Q}} \frac{\left|x-x_{0}\right|}{\left|x_{0}-y\right|^{n+1-\delta}}|f(z)| d z \\
\leq & C \sum_{|\alpha|=m}\left\|D^{\alpha} A\right\|_{\dot{\wedge}_{\beta}|Q|^{\beta / n}} M_{\delta, 1}(f)(\tilde{x}) .
\end{aligned}
$$


For $I I I_{3}$, by the inequality: $a^{1 / 2}-b^{1 / 2} \leq(a-b)^{1 / 2}$ for $a \geq b>0$, we gain, similar to the proof of Lemma 6,

$$
\begin{aligned}
& \frac{1}{|Q|^{1+\beta / n}} \int_{Q}\left\|I I I_{3}\right\| d x \\
\leq & \frac{C}{|Q|^{1+\beta / n}} \int_{Q} \int_{R^{n}} \\
& \left(\int_{R_{+}^{n+1}}\left[\frac{t^{n \mu / 2}\left|x-x_{0}\right|^{1 / 2}\left|\psi_{t}(y-z)\right|\left|R_{m}\left(\tilde{A} ; x_{0}, z\right) \| f_{2}(z)\right|}{|x-z|^{m}(t+|x-y|)^{(n \mu+1) / 2}}\right]^{2} \frac{d y d t}{t^{n+1}}\right)^{1 / 2} d z d x \\
\leq & \frac{C}{|Q|^{1+\beta / n}} \int_{Q} \int_{R^{n}} \frac{\left|f_{2}(z) \| R_{m}\left(\tilde{A} ; x_{0}, z\right)\right|\left|x-x_{0}\right|^{1 / 2}}{|x-z|^{m}} \\
& \times\left(\int_{0}^{\infty} \frac{d t}{(t+|x-z|)^{2 n+2-2 \delta}}\right)^{1 / 2} d z d x \\
\leq & C \sum_{|\alpha|=m}\left\|D^{\alpha} A\right\|_{\lambda_{\beta}} \int_{R^{n}} \frac{\left|x-x_{0}\right|^{1 / 2}}{\left|x_{0}-z\right|^{n+1 / 2-\delta}\left|f_{2}(z)\right| d z} \\
\leq & C \sum_{|\alpha|=m}\left\|D^{\alpha} A\right\|_{\lambda_{\beta}} \sum_{k=1}^{\infty} 2^{-k / 2} M_{\delta, 1}(f)(\tilde{x}) \leq C \sum_{|\alpha|=m}\left\|D^{\alpha} A\right\| \|_{\lambda_{\beta}} M_{\delta, 1}(f)(\tilde{x}) .
\end{aligned}
$$

For $\mathrm{III}_{4}$, by Lemma 4, we get

$$
\left|D^{\alpha} A(z)-\left(D^{\alpha} A\right)_{\tilde{Q}}\right| \leq\left.|| D^{\alpha} A\right|_{\dot{\lambda}_{\beta}}\left|x_{0}-z\right|^{\beta},
$$

thus, similar to the proof of $I I I_{1}$ and $I I I_{3}$, we obtain

$$
\begin{aligned}
& \frac{1}{|Q|^{1+\beta / n}} \int_{Q}\left\|I I I_{4}\right\| d x \\
\leq & \frac{C}{|Q|^{1+\beta / n}} \int_{Q} \sum_{|\alpha|=m} \int_{R^{n}}\left(\frac{\left|x-x_{0}\right|}{\left|x_{0}-z\right|^{n+1-\delta}}+\frac{\left|x-x_{0}\right|^{1 / 2}}{\left|x_{0}-z\right|^{n+1 / 2-\delta}}\right)\left|f_{2}(z) \| D^{\alpha} \tilde{A}(z)\right| d z d x \\
\leq & C \sum_{|\alpha|=m}\left\|D^{\alpha} A\right\|_{\lambda_{\beta}} \sum_{k=1}^{\infty}\left(2^{k(\beta-1)}+2^{k(\beta-1 / 2)}\right) M_{\delta, 1}(f)(\tilde{x}) \\
\leq & C \sum_{|\alpha|=m}\left\|D^{\alpha} A\right\|_{\lambda_{\beta}} M_{\delta, 1}(f)(\tilde{x}) .
\end{aligned}
$$

Thus,

$$
I I I \leq C \sum_{|\alpha|=m}\left\|D^{\alpha} A\right\|_{{\dot{\lambda_{\beta}}}} M_{\delta, 1}(f)(\tilde{x}) .
$$

We now put these estimates together, and taking the supremum over all $Q$ such that $\tilde{x} \in Q$, and using Lemma 1 with Lemma 3 , we obtain

$$
\left\|g_{\mu}^{A}(f)\right\|_{\dot{F}_{q}^{\beta, \infty}} \leq\left. C \sum_{|\alpha|=m}\left\|D^{\alpha} A\right\|\right|_{\dot{\lambda}_{\beta}}|| f \|_{L^{p}} .
$$

This completes the proof of Theorem 1 . 
Proof of Theorem 2. By the same argument as in the proof of Theorem 1, we have, for $1 \leq s<p$ and $1 / r=1 / s-\delta / n$,

$$
\begin{aligned}
& \frac{1}{|Q|} \int_{Q}\left|g_{\mu}^{A}(f)(x)-g_{\mu}^{\tilde{A}}\left(f_{2}\right)\left(x_{0}\right)\right| d x \\
\leq & C \sum_{|\alpha|=m}\left\|D^{\alpha} A\right\|_{{\dot{\aleph_{\beta}}}_{\beta}}\left(M_{\beta+\delta, r}(f)+M_{\beta+\delta, 1}(f)\right),
\end{aligned}
$$

thus, the sharp estimate of $g_{\mu}^{A}$ is obtained as following

$$
\left(g_{\mu}^{A}(f)\right)^{\#} \leq C \sum_{|\alpha|=m}\left\|D^{\alpha} A\right\|_{\dot{\lambda}_{\beta}}\left(M_{\beta+\delta, r}(f)+M_{\beta+\delta, 1}(f)\right)
$$

Now, using Lemma 3, we gain

$$
\begin{aligned}
\left\|g_{\mu}^{A}(f)\right\|_{L^{q}} & \leq C\left\|\left(g_{\mu}^{A}(f)\right)^{\#}\right\|_{L^{q}} \\
& \leq C \sum_{|\alpha|=m}\left\|D^{\alpha} A\right\|_{\dot{\lambda}_{\beta}}\left(\left\|M_{\beta+\delta, r}(f)\right\|_{L^{q}}+\left\|M_{\beta+\delta, 1}(f)\right\|_{L^{q}}\right) \leq C\|f\|_{p} .
\end{aligned}
$$

This completes the proof of Theorem 2 .

Proof of Theorem 3. We first prove the following estimate:

$$
\left|g_{\mu}^{A}(f)(x)\right| \leq C \sum_{|\alpha|=m}\left\|D^{\alpha} A\right\|_{\dot{\lambda}_{\beta}}\left(\lambda_{1}^{\delta+\beta} M f(x)+\lambda_{1}^{\delta+\beta-n / r^{\prime}}\|f\|_{L^{1}}^{1 / r^{\prime}}(M f(x))^{1 / r}\right)
$$

for any $\lambda_{1}>0$ and $n /(n-\delta-\beta)<r$. In fact, fix the cube $Q=Q\left(x, \lambda_{1}\right)$, similar to the proof of Lemma 6 , we have

$$
\begin{aligned}
\left|g_{\mu}^{A}(f)(x)\right| & \leq C \int_{R^{n}} \frac{|f(z)|\left|R_{m+1}(A ; x, z)\right|}{|x-z|^{m+n-\delta}} d z \\
& =C\left(\int_{Q}+\int_{Q^{c}}\right) \frac{|f(z)|\left|R_{m+1}(A ; x, z)\right|}{|x-z|^{m+n-\delta}} d z=I_{1}+I_{2} .
\end{aligned}
$$

For $I_{1}$, we let that, for $k>0$,

$$
\tilde{A}_{k}(y)=A(y)-\sum_{|\alpha|=m} \frac{1}{\alpha !}\left(D^{\alpha} A\right)_{2^{-k} Q} y^{\alpha}
$$

then, by Lemma 5 , for $z \in 2^{-k} Q$,

$$
\left|R_{m+1}\left(\tilde{A}_{k} ; x, z\right)\right| \leq C \sum_{|\alpha|=m}\left\|D^{\alpha} A\right\|_{\dot{\lambda}_{\beta}}\left(2^{-k} \lambda_{1}\right)^{\beta}|x-z|^{m}
$$


thus, by Lemma 4 and Lemma 5 ,

$$
\begin{aligned}
I_{1} & \leq C \sum_{k=0}^{\infty} \int_{2^{-k} Q \backslash 2^{-k-1} Q} \frac{|f(z)|\left|R_{m+1}\left(\tilde{A}_{k} ; x, z\right)\right|}{|x-z|^{m+n-\delta}} d z \\
& \leq C \sum_{|\alpha|=m}\left\|D^{\alpha} A\right\|{\dot{\lambda_{\beta}}}_{k_{k=0}}^{\infty}\left(2^{-k} \lambda_{1}\right)^{\beta} \int_{2^{-k} Q \backslash 2^{-k-1} Q} \frac{|f(z)|}{|x-z|^{n-\delta}} d z \\
& \leq C \sum_{|\alpha|=m}\left\|D^{\alpha} A\right\|_{\dot{\lambda}_{\beta}} \sum_{k=0}^{\infty}\left(2^{-k} \lambda_{1}\right)^{\beta+\delta-n} \int_{2^{-k} Q \backslash 2^{-k-1} Q}|f(z)| d z \\
& \leq\left. C \sum_{|\alpha|=m}\left\|D^{\alpha} A\right\|\right|_{\dot{\lambda}_{\beta}} \lambda_{1}^{\beta+\delta} M f(x) ;
\end{aligned}
$$

For $I_{2}$, taking $\varepsilon>0$ such that $(n+\varepsilon) /(n-\delta-\beta)<r$, we write $n-\delta=$ $(n+\varepsilon) / r+n / r^{\prime}-\varepsilon / r-\delta$, then, by the Hölder's inequality,

$$
\begin{aligned}
I_{2} \leq & C\left(\int_{Q^{c}} \frac{|f(z)| d z}{|x-z|^{n+\varepsilon}}\right)^{1 / r}\left[\int_{Q^{c}} \frac{|f(z)|}{|x-z|^{n-(\delta+\varepsilon / r) r^{\prime}}}\left(\frac{\left|R_{m+1}(A ; x, z)\right|}{|x-z|^{m}}\right)^{r^{\prime}} d z\right]^{1 / r^{\prime}} \\
\leq & C \sum_{|\alpha|=m}\left\|D^{\alpha} A\right\|_{\dot{\lambda}_{\beta}}\left(\sum_{k=0}^{\infty}\left(2^{k} \lambda_{1}\right)^{-\varepsilon-n} \int_{|x-z|<2^{k} \lambda_{1}}|f(z)| d z\right)^{1 / r} \\
& \times\left(\sum_{k=0}^{\infty}\left(2^{k} \lambda_{1}\right)^{\beta r^{\prime}} \int_{2^{-k} Q \backslash 2^{-k-1} Q} \frac{|f(z)| d z}{|x-z|^{n-(\delta+\varepsilon / r) r^{\prime}}}\right)^{1 / r} \lambda_{1}^{\delta+\beta-n / r^{\prime}+\varepsilon / r} \\
\leq & C \sum_{|\alpha|=m}\left\|D^{\alpha} A\right\|_{\dot{\lambda}_{\beta}}\left(\sum_{k=0}^{\infty} 2^{-k \varepsilon} \lambda_{1}^{-\varepsilon} M f(x)\right)^{1 / r^{\prime}} \\
& \times\left(\sum_{k=0}^{\infty} 2^{k\left(\delta+\beta-n / r^{\prime}+\varepsilon / r\right) r^{\prime}} \int_{2^{-k} Q \backslash 2^{-k-1} Q}^{|f(z)| d z}\right)^{\leq} \\
& \left.C \sum_{|\alpha|=m}\left\|D^{\alpha} A\right\|\right|_{\dot{\lambda}_{\beta}} \lambda_{1}^{-\varepsilon / r}(M f(x))^{1 / r} \lambda_{1}^{\delta+\beta-n / r^{\prime}+\varepsilon / r}\|f\|_{L^{1}}^{1 / r^{\prime}} \\
\leq & C \sum_{|\alpha|=m}\left\|D^{\alpha} A\right\|{\dot{\lambda_{\beta}}}_{\beta} \lambda_{1}^{\delta+\beta-n / r^{\prime}}\|f\|_{L^{1}}^{1 / r^{\prime}}(M f(x))^{1 / r} .
\end{aligned}
$$

Thus, our claim holds. Now we can prove Theorem 3. For any $\lambda>0$ and $f \in L^{1}\left(R^{n}\right)$, taking $\lambda_{1}=\left(\sum_{|\alpha|=m}\left\|D^{\alpha} A\right\|_{\dot{\lambda}_{\beta}}\|f\|_{L^{1}} \lambda^{-1}\right)^{1 /(n-\delta-\beta)}$ in above estimate, 
we gain, by the weak type boundedness of $M$,

$$
\begin{aligned}
& \left|\left\{x \in R^{n}: g_{\mu}^{A}(f)(x)>\lambda\right\}\right| \\
\leq & \left|\left\{x \in R^{n}: M f(x)>\frac{\lambda}{2 C \sum_{|\alpha|=m}\left\|D^{\alpha} A\right\|_{\dot{\lambda}_{\beta}} \lambda_{1}^{\delta+\beta}}\right\}\right| \\
& +\left|\left\{x \in R^{n}: M f(x)>\left(\frac{\lambda}{2 C \sum_{|\alpha|=m}|| D^{\alpha} A\left\|_{\dot{\lambda}_{\beta}} \lambda_{1}^{\delta+\beta-n / r^{\prime}}\right\| f \|_{L^{1}}^{1 / r}}\right)^{r}\right\}\right| \\
\leq & C \sum_{|\alpha|=m}\left\|D^{\alpha} A\right\|_{\dot{\lambda}_{\beta}} \lambda_{1}^{\delta+\beta}\|f\|_{L^{1}} / \lambda+C\left(\sum_{|\alpha|=m}\left\|D^{\alpha} A\right\|_{\dot{\lambda}_{\beta}} \lambda_{1}^{\delta+\beta-n / r^{\prime}}\|f\|_{L^{1}}^{1 / r} / \lambda\right)^{r} \\
\leq & C\left(\sum_{|\alpha|=m}\left\|D^{\alpha} A\right\|_{\dot{\lambda}_{\beta}}\|f\|_{L^{1}} / \lambda\right)^{n /(n-\delta-\beta)}
\end{aligned}
$$

This completes the proof of Theorem 3 .

Acknowledgement. The author would like to express his gratitude to the referee for his very valuable comments and suggestions.

\section{REFERENCES}

[1] J. Alvarez, R. J. Babgy, D. S. Kurtz and C. Perez, Weighted estimates for commutators of linear operators, Studia Math., 104 (1993), pp. 195-209.

[2] S. Chanillo, A note on commutators, Indiana Univ. Math. J., 31 (1982), pp. 7-16.

[3] W. G. Chen, Besov estimates for a class of multilinear singular integrals, Acta Math. Sinica, 16 (2000), pp. 613-626.

[4] J. CoHEN, A sharp estimate for a multilinear singular integral on $R^{n}$, Indiana Univ. Math. J., 30 (1981), pp. 693-702.

[5] J. Cohen And J. Gosselin, On multilinear singular integral operators on $R^{n}$, Studia Math., 72 (1982), pp. 199-223.

[6] J. Cohen And J. Gosselin, A BMO estimate for multilinear singular integral operators, Illinois J. Math., 30 (1986), pp. 445-465.

[7] R. Coifman, R. Rochberg and G. Weiss, Factorization theorems for Hardy spaces in several variables, Ann. of Math., 103 (1976), pp. 611-635.

[8] R. A. DeVore and R. C. Sharpley, Maximal functions measuring smoothness, Mem. Amer. Math. Soc., 47 (1984).

[9] Y. Ding And S. Z. Lu, Weighted boundedness for a class rough multilinear operators, Acta Math. Sinica, 3 (2001), pp. 517-526.

[10] G. Hu AND D. C. YANG, A variant sharp estimate for multilinear singular integral operators, Studia Math., 141 (2000), pp. 25-42.

[11] S. JANson, Mean oscillation and commutators of singular integral operators, Ark. Math., 16 (1978), pp. 263-270.

[12] S. Janson, M. Taibleson and G. Weiss, Elementary characterizations of the MorreyCampanato spaces, Lect. Notes in Math., 992 (1983), pp. 101-114.

[13] Liu Lanzhe, Continuity for commutators of Littlewood-Paley operators on certain Hardy spaces, J. of the Korean Math. Soc., 40 (2003), pp. 41-60.

[14] Liu Lanzhe, Weighted weak type estimates for commutators of Littlewood-Paley operator, Japanese J. of Math., 29:1 (2003), pp. 1-13.

[15] Liu Lanzhe, Boundedness for commutators of Littlewood-Paley operators on some Hardy spaces, Lobachevskii J. of Math., 12 (2003), pp. 63-71.

[16] LiU Lanzhe, Triebel-Lizorkin spaces estimates for multilinear operators of sublinear operators, Proc. Indian Acad. Sci. (Math.Sci.), 113 (2003), pp. 379-393.

[17] M. Paluszynski, Characterization of the Besov spaces via the commutator operator of Coifman, Rochberg and Weiss, Indiana Univ. Math. J., 44 (1995), pp. 1-17. 
[18] A. Tonchinsky, The real variable methods in harmonic analysis, Pure and Applied Math. 123, Academic Press, New York, 1986. 
L. LIU 